\title{
Impact on the Quality of Life of an Educational Program for the Prevention of Work-Related Musculoskeletal Disorders: a randomized controlled trial
}

\author{
Antonio C Santos*, Markus Bredemeier, Karen F Rosa, Vinicius A Amantéa, Ricardo M Xavier
}

\begin{abstract}
Background: Work-related musculoskeletal disorders (WMSD) are a major cause for concern in public health and the main causes of sick leave. Treatments for WMSD have given disappointing results; prevention is the best strategy, but results of preventive measures have not been consistent. To the best of our knowledge there are few studies in literature that evaluated the impact of a specific program aimed at preventing WMSD on the quality of life of employed persons.

Methods: One hundred and one clerical and production workers in a steel trading company were enrolled in an open-label randomized controlled clinical trial (parallel groups) to compare the efficacy of an educational program for primary prevention of WMSD with control intervention. The primary outcome was a change in the physical functioning domain of the quality of life (QL) measured by Medical Outcomes Study Short Form 36 Health Survey (SF-36). The intervention group underwent six consecutive weekly sessions concerning specific orientations for the prevention of WMSD, while the control group received general health education in an identical schedule. The SF-36 and theses Work Limitation Questionnaire (WLQ) were evaluated at weeks zero, five and 26.
\end{abstract}

Results: Baseline characteristics of the interventions groups were comparable, and both groups comprised predominantly young healthy individuals. No significant differences in the variation of the SF-36 and WLQ between the groups were observed at weeks five and 26. However, both groups demonstrated improvement in some aspects of SF-36, suggesting that both educational interventions have beneficial impacts on QL.

Conclusions: A specific educational program aimed at the preventing of WMSD was comparable with general health orientation for the improvement of QL and work capacity in a sample of healthy workers during a six month period.

Trial Registration: ClinicalTrials.gov: NCT00874718

Trial Registration

\section{Background}

Work-related musculoskeletal disorders (WMSD) are a major concern for public health and frequently lead to temporary or permanent work incapacity $[1,2]$. The etiology of WMSD is complex, and includes ergonomic, individual, psychological and social factors [3-5]. In the

\footnotetext{
* Correspondence: acsantos@hcpa.ufrgs.br

Hospital de Clínicas de Porto Alegre, Universidade Federal do Rio Grande do Sul, R. Ramiro Barcelos, 2350, Porto Alegre, 90035-903, Rio Grande do Sul, Brazil
}

(C) 2011 Santos et al; licensee BioMed Central Ltd. This is an Open Access article distributed under the terms of the Creative Commons

USA, 4.26 million nonfatal work-related injuries and illnesses were reported in private industry during 2004, representing an incidence of 4.8 cases per 100 equivalent full-time workers. A significant portion of all events (1.26 million, 29.6\%) were related to days spent away from work, and approximately 0.4 million of these were attributed to musculoskeletal disorders [1]. In specific working populations, the prevalence of WMSD can be as high as $22-40 \%$, according to a recent review [6]. In a cohort study concerning industrial and clerical 
American workers the cumulative incidence rate of upper extremity tendonitis was $24.3 \%$ during an average follow-up of 5.4 years [7]. In Brazil, accurate statistical data are scant, but according to the National Institute of Social Security (INSS), WMSD is the second most frequent cause of sick leave [2].

Treatment for WMSD has frequently shown disappointing results and prevention is regarded as the best strategy to avoid economic and social consequences [6,8-11]. Interventional preventive measures have been tested in randomized controlled trials, but results have been mixed [12-20], and the effectiveness of these programs has not been consistent. This variability could be related to the type of work, differences in training and/or educational programs, and methodological issues related to study design.

To our knowledge, no previous study has evaluated the effect of a specific program aimed at the prevention of WMSD on the quality of life (QL). It may be the case that an effective educational program could improve QL for workers through teaching techniques of avoidance and controlling risk factors for WMSD. Therefore, we designed a randomized controlled trial to evaluate the impact of a specific educational program for the prevention of WMSD on measures of QL, particularly physical functioning, on a sample of workers from a steel trading company.

\section{Methods}

\section{Study Design}

This was an open label randomized controlled trial of parallel groups comparing the effect on QL of a specific educational program designed for the prevention of WMSD versus a general health orientation program.

\section{The study population, recruitment and randomization}

The study population included clerical staff and production workers from a steel trading company located in Porto Alegre/RS/Brazil (Aços Favorit Ltda TM). All workers were involved in a routine of overcharge tasks and/or repetitive movements. The company had a total of 131 active employees (May/2008). Workers less than 18 years old, on sick leave, working night shift, on vacation or those that could not be released from their activities (most of these were supervisors), were excluded. One hundred and twenty employees were eligible for randomization, and 101 agreed to participate in the study.

The company presented two lists of workers organized in alphabetic order (one list consisting of clerical workers and other consisting of production workers). Using WINPEPI software [21], both lists were reorganized in an aleatory sequence, and the workers were invited to participate following this sequence. Individuals that were considered eligible for randomization (after written informed consent) were included in a numbered sequential list for randomization. Therefore, two numerically ordered lists (one for clerical and other for production workers) were produced. Using the WINPEPI software, the workers within each list were randomly allocated to one of two groups (intervention or control group) (Figure 1).

\section{Interventions}

Subjects allocated to the intervention group attended six consecutive weekly training sessions of one hour duration at the worksite (during the working period) administered by the main investigator (ACS). The number of participants in each session was limited to 25 individuals [18], and the structure of "group dynamics" (involving interaction, discussion, dramatization of daily living and work related activities) was used. The educational program was applied using flip-charts and printed material (no computer-based resources were used during the sessions). The training sessions followed the schedule as described below:

- First meeting: discussion of the importance of work for QL. Debate about the objectives and elements of the educational program for prevention of WMSD, the body as a major work tool, work-related and daily living overload of the musculoskeletal system, and the importance and duration of breaks. Demonstration of some specific stretching exercises for the neck and upper extremity muscles. Distribution of didactic material (to be read as homework) reinforcing the issues discussed in the first meeting and introducing the topics of the next meeting.

- Second meeting: review of issues discussed in the previous meeting. Demonstration and practice of breaks. Discussion of psychological and emotional stress. Orientation for the identification of risk situations for WMSD in specific task jobs. Distribution of didactic material concerning strategies to identify and control stress, and pictures concerning overload tasks and bad postures.

- Third meeting: discussion concerning the main objectives of ergonomics, bad postures, and overload tasks. Debate about stress and interpersonal relationships. Distribution of didactic material for the fourth and fifth meetings.

- Fourth meeting: an interactive training with the aim to stimulating an attitude in which each worker observes and warns his colleagues (subgroups consisting of two or three individuals) about breaks, relaxation, stretching exercises, and good posture.

- Fifth meeting: debate of physiological responses to emotional stress. Training in exercises for relaxation 


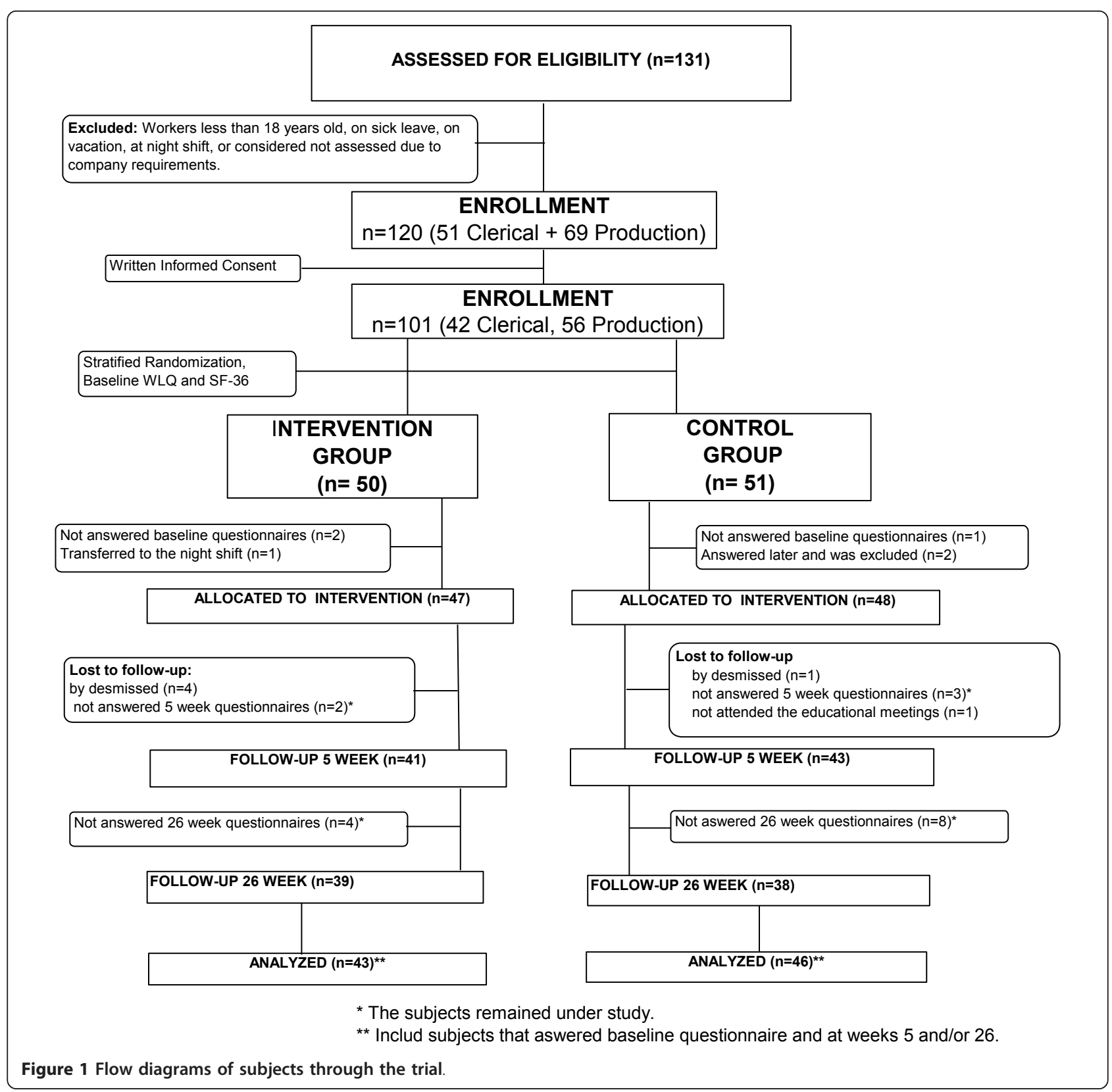

and stretching of the upper extremities, neck, lower back and lower extremities.

- Sixth meeting: review of the subjects discussed in previous meetings and general health orientations concerning sleep, alimentation, hygiene, physical activities and well-being.

Individuals allocated to the control group participated in six consecutive weekly meetings of one hour duration at the worksite, during working hours, and up to 25 individuals participated in each meeting. The entire sequence of control group meetings began in the week following the end of the series of intervention group sessions. Both groups were conducted in same season (winter). The educational program was delivered by a general practitioner (KFR) using "group dynamics" techniques (involving interaction, discussion, dramatization of daily living and work related activities) with flipcharts and printed material (no computer-based resources were used during the sessions). This program consisted of explanations and debates about important general health themes, as described below. 
- First meeting: discussion concerning nutrition and avoiding obesity.

- Second meeting: techniques for the control and reduction of stress.

- Third meeting: hygiene and prevention of diseases.

- Fourth meeting: the importance of adequate sleep and practices to reduce insomnia.

- Fifth and sixth meetings: changing lifestyle and tips for a safe and healthy life.

For both study groups, the themes discussed in the meetings were reinforced through the distribution of new didactic material three and five months after the end of the educational programs.

\section{Measurements}

Demographic and work-related data for all workers were obtained and recorded at entry into the study. The physical functioning domain measured by the Medical Outcomes Study Short Form 36 Health Survey (SF-36) was the primary outcome. This questionnaire is a general, widely used tool to evaluate QL, with eight scales: physical functioning, role physical, bodily pain, general health, vitality, social functioning, role emotional, mental health, and two summaries: the physical component and the mental component. The scales ranges from 0 (worst condition) up to 100 (best condition) [22]. The secondary outcomes were the other domains of SF-36 and the work capacity evaluated by the Work Limitation Questionnaire [WLQ]. WLQ is a tool with four scales: time management, physical demands, mental-interpersonal demands, output demands and a percentage index. The scales ranges from 0 (without limitation) up to 100 (limitations all the time) and it is possible to calculate a WLQ index using a table to define the loss of productivity. For example a 5.0 WLQ index represents a $4.9 \%$ loss in productivity when compared with healthy people [23]. Both questionnaires, the SF-36 and WLQ were well designed and easy to administer, and validated for the Brazilian-Portuguese language [22,23]. The questionnaires were self-administered before the interventions and five and 26 weeks after completion of the educational programs.

\section{Sample size calculation}

Estimating that a difference of 10 points in variation of the physical functioning SF-36 score would be clinically relevant, and considering a standard deviation of 14.0 for this variation in both groups [24], 32 patients in each experimental group would provide $80.0 \%$ statistical power to detect a significant difference $(\mathrm{P} \leq 0.05)$. However, considering the possibility of loss to follow up of some individuals, a total of 101 employees underwent stratified randomization.

\section{Ethical aspects}

After being invited to participate and accepting, workers signed a written informed consent before randomization. The study project was approved by the Research Ethics Committee of the Universidade Federal do Rio Grande do Sul (registration number: 2007755). The study was also registered at ClinicalTrials.gov (number NCT00874718).

\section{Statistical Methods}

The data were analyzed using Epi Info, version 6 and SPSS for Windows, version 14.0. Quantitative variables were graphically and statistically tested (with the Kolmogorov-Smirnov goodness-of-fit test) for normality of distribution. Student's $t$-test was used to evaluate differences in the primary and secondary outcomes. Other variables with a normal distribution were presented as the mean $\pm \mathrm{SD}$, and the intra-group and inter-group comparisons were performed using paired and unpaired Student's $t$-tests, respectively. Baseline non-normal quantitative variables were presented as the median (25th, 75 th percentiles). A chi-square goodness-of-fit test was used to compare observed and expected frequencies of sick-leaves. A two-tailed $P$ value less than or equal to 0.05 was considered statistically significant.

\section{Results}

Of 50 patients allocated to the intervention group, two did not fill in baseline questionnaires, one was transferred to the night shift, and four were dismissed. Among the 51 patients allocated to control group, three did not fill in baseline questionnaires, one did not attend the educational meetings, and one was dismissed. The demographic and work-related features of the subjects included in the analysis are presented in Table 1.

Table 1 demonstrates that both groups had a similar distribution of age, gender, race, marital status, sector of job, medical condition, and current use of medications, body mass index, physical activity and years in work. The intervention group tended to have a higher median baseline physical functioning (table 1) and bodily pain score of SF-36 (median, percentiles 25-75: 72.0, $51.0-84.0$ versus $61.5,41.0-84$ in controls; $\mathrm{P}=0.043$ ).

\section{Primary analysis}

At week 26 no significant differences in the variation of the physical functioning domain of the SF-36 were evident: -1.49 in the intervention group and 0.92 in the control group, mean difference of -2.41 ( $95 \%$ confidence interval, -11.8, 2.32) (Table 2). The results remained comparable when clerical and production workers were analyzed separately. 
Table 1 Demographic and work-related characteristics of the study subjects*

\begin{tabular}{|c|c|c|}
\hline & Intervention Group ( $n=43$ ) & Control Group $(n=46)$ \\
\hline Age - mean (SD) & $30.6(10.2)$ & $31.2(8.8)$ \\
\hline Male & $26(60.5)$ & $34(73.9)$ \\
\hline Caucasians & $36(83.7)$ & $42(91.3)$ \\
\hline Married & $20(46.5)$ & $26(56.5)$ \\
\hline \multicolumn{3}{|l|}{ Educational status t: } \\
\hline complete ES & $2(5.4)$ & $6(14.3)$ \\
\hline complete HS & $32(86.5)$ & $32(76.2)$ \\
\hline complete university & $3(8.1)$ & $4(9.5)$ \\
\hline \multicolumn{3}{|l|}{ Sector: } \\
\hline administrative & $20(46.5)$ & $18(39.1)$ \\
\hline productive & $23(53.5)$ & $28(60.9)$ \\
\hline Medical condition & $7(16.3)$ & $12(26.1)$ \\
\hline Current use of medications & $7(16.3)$ & $7(15.2)$ \\
\hline Body Mass Index - mean (SD) & $25.0(3.0)$ & $26.0(2.9)$ \\
\hline Physical activity & $26(63.4)$ & $28(60.9)$ \\
\hline Years of work - median (percentiles 25,75 ) & $7.0(3.0,14.0)$ & $10.5(3.88,19.0)$ \\
\hline Number of presences in the educational meetings - median (percentiles 25,75 ) & $6.0(5.0,6.0)$ & $6.0(5.0,6.0)$ \\
\hline Baseline Physical functioning score - median (percentiles 25, 75) & $95.0(85.0,100.0)$ & $90.0(85.0,95.0)$ \\
\hline Baseline Work limitation questionnaire index (percent) - median (percentiles 25,75 ) & $2.76(1.67,3.63)$ & $2.88(1.10,5.03)$ \\
\hline
\end{tabular}

Table 2 Comparison of the variation of the SF-36 and WLQ scores (between baseline and week 26) in the experimental groups

\begin{tabular}{|c|c|c|c|c|c|c|c|}
\hline & \multicolumn{2}{|c|}{ Intervention Group } & \multicolumn{2}{|c|}{ Control Group } & \multirow[t]{2}{*}{ Mean difference } & \multirow[t]{2}{*}{$95 \% \mathrm{Cl}$} & \multirow[t]{2}{*}{ P Value ${ }^{*}$} \\
\hline & $\mathrm{n}$ & Mean (SD) & $\mathbf{n}$ & Mean (SD) & & & \\
\hline \multicolumn{8}{|c|}{ SF-36 scores } \\
\hline Physical functioning & 37 & $-1.49(21.5)$ & 38 & $0.92(19.4)$ & -2.41 & $-11.8,7.0$ & 0.613 \\
\hline Role physical & 38 & $6.58(24.4)$ & 38 & 5.49 (34.6) & 1.09 & $-12.6,14.8$ & 0.874 \\
\hline Bodily pain & 39 & $10.9(21.1) \dagger$ & 38 & $9.63(22.4) \dagger$ & 1.27 & $-8.6,11.2$ & 0.800 \\
\hline General health & 36 & $4.94(10.4) \dagger$ & 36 & $2.64(13.9)$ & $2 ; 31$ & $-3.5,8.1$ & 0.428 \\
\hline Vitality & 36 & $7.78(16.2) \dagger$ & 37 & $6.76(20.2)$ & 1.02 & $-7.5,9.6$ & 0.813 \\
\hline Social functioning & 35 & $6.43(21.9)$ & 36 & $11.11(23.9) \dagger$ & -4.68 & $-15.5,6.2$ & 0.393 \\
\hline Role emotional & 38 & 7.89 (39.8) & 38 & $14.04(35.2)$ & -6.14 & $-23.3,11.0$ & 0.479 \\
\hline Mental health & 39 & $8.37(16.0) \dagger$ & 37 & $4.32(16.5)$ & 4.05 & $-3.4,11.5$ & 0.281 \\
\hline Physical component summary & 34 & $5.04(10.1) \dagger$ & 36 & $4.64(16.2)$ & 0.41 & $-6.1,6.9$ & 0.900 \\
\hline Mental component summary & 31 & $8.55(17.2) \dagger$ & 34 & $9.52(19.4) \dagger$ & -0.97 & $-10.1,8.1$ & 0.832 \\
\hline \multicolumn{8}{|c|}{ WLQ scores } \\
\hline Time management & 36 & $0.56(13.5)$ & 35 & $-0.11(14.3)$ & 0.66 & $-5.9,7.2$ & 0.841 \\
\hline Physical demands & 37 & $4.62(32.5)$ & 38 & $3.35(38.3)$ & 1.27 & $-15.1,17.6$ & 0.877 \\
\hline Mental-interpersonal demands & 37 & $-2.10(6.3)$ & 37 & $-1.70(11.6)$ & -0.41 & $-4.8,3.9$ & 0.853 \\
\hline Output demands & 38 & $-0.92(9.4)$ & 37 & $-5.95(12.8) \dagger$ & 5.02 & $-0.2,10.2$ & 0.057 \\
\hline WLQ index (percent) & 34 & $-0.18(2.4)$ & 34 & $-0.72(3.2)$ & 0.54 & $-0.8,1.8$ & 0.435 \\
\hline
\end{tabular}

* Student's $t$ test. $+\mathrm{P} \leq 0.01$ by paired Student's $t$ comparing baseline and week 26 scores within the group.

Abbreviations: SF-36 = Medical Outcomes Study 36 - Item short-form health survey; WLQ = Work Limitation Questionnaire. 


\section{Secondary analysis}

No significant differences between the intervention and control groups were observed in the secondary outcomes (Table 2). The intra-group variations of the SF-36 and WLQ scores showed significant improvements in bodily pain scores, general health, vitality, mental health, Physical Component Summary (PCS), and Mental Component Summary (MCS) in the intervention group (Table 2). The control group presented statistically significant improvements in bodily pain, social functioning, MCS and output demands (Table 2).

In this study, the incidence of WMSD with days away from work was very low during, before and after the study period. In the 10 months prior to the study, during the three months of the educational program, and in the seven months after completion, there were only four, one and one occurrences, respectively (goodnessof-fit test, $\mathrm{P}=0.631$ ).

\section{Discussion}

The present study was designed to compare the effects on QL measured by SF-36, of a specific educational program aimed at preventing WMSD and a program of general health orientation, in workers from a steel trading company. No significant differences in the outcomes were observed between the groups. However, both groups presented with improvements in some physical and emotional aspects.

Previous RTC studies evaluating interventions for the prevention of WMSD have yielded inconsistent results. This may be related to differences in the kinds of jobs and tasks, the focus on different WMSDs, differences in the prevention programs, and methodological issues, making comparisons between studies difficult. Andersen et al [25], compared the effect of specific exercises training, general exercises, and general health counseling in office workers, and demonstrated reductions in neck and shoulder pain with both types of exercises, but no effect of general health education. In a reanalysis of the same data, the authors observed that asymptomatic individuals at the baseline were more likely to be free of neck-shoulder pain when allocated to the specific training group [26]. Two studies that tested interventions for the prevention of back pain using a back school based education program in the occupational setting (post office) [12,14] and companies involving physically demanding jobs [14] in comparison to usual care, demonstrated no benefit from a training program. Pillastrini et al compared the effect of an informative brochure (concerning self-care at the work station) and ergonomic intervention with an informative brochure alone for video display terminal users. In the group that received the ergonomic intervention there was evidence of lower overload in tasks, and a reduction in lower back, neck, and shoulder symptoms [15].

In the present study, the absence of significant differences between the intervention and control groups may be due to several possible factors. Both groups received educational health orientations and subjects allocated to different groups were working in the same environment, so it is possible that the motivation for the prevention of WMSD also increased in the control group by "contamination" from the experimental group. The observation that both groups demonstrated significant improvements in some aspects of the SF36 and WLQ may be related to a placebo effect and/or the fact of being part of a clinical trial ("Hawthorne effect") [27]. Additionally, the orientation about a health promoting behavior in the control group may have had a beneficial effect on QL. Given that the final evaluation was performed at six months, an effect of intervention in the long term cannot be ruled out. In addition, considering that the differences between the intervention and control groups are not clinically or statistically significant (and no consistent numerical trend in favor of one groups was evident), lack of statistical power is not a likely explanation. However, some confidence intervals were wide (some including a difference of 10 points in the variation of scores) and a clinically significant difference between the groups cannot be excluded.

Another possible explanation for the absence of a difference in results is the "ceiling effect", which occurs when the preventing effect is diluted by the good health condition of the sample under study. The company where the study took place had a low incidence of sickleave, and the mean age and the mean number of years of labor activity in the sample were relatively low. The mean baseline values of all domains of the SF-36 and the WLQ were within the normal range. In addition, baseline values in the control group for the domains of physical functioning (table 1) and bodily pain of the SF36 were worse than in the experimental group. Furthermore, a negative correlation was observed between the baseline physical functioning score and variation of this score between baseline and week 26 (figure 2). Therefore, subjects with lower baseline scores presented with significantly greater improvements at the 26 week evaluation. It is possible that the control group was subjected to a more intense "regression to the mean" effect, thus reducing differences with the intervention group.

\section{Conclusion}

This study failed to demonstrate a beneficial impact on the quality of life of an educational program aimed to prevent WMSD in comparison with general health education, in a sample of healthy workers with a low incidence of sick-leave. However, both groups demonstrated 


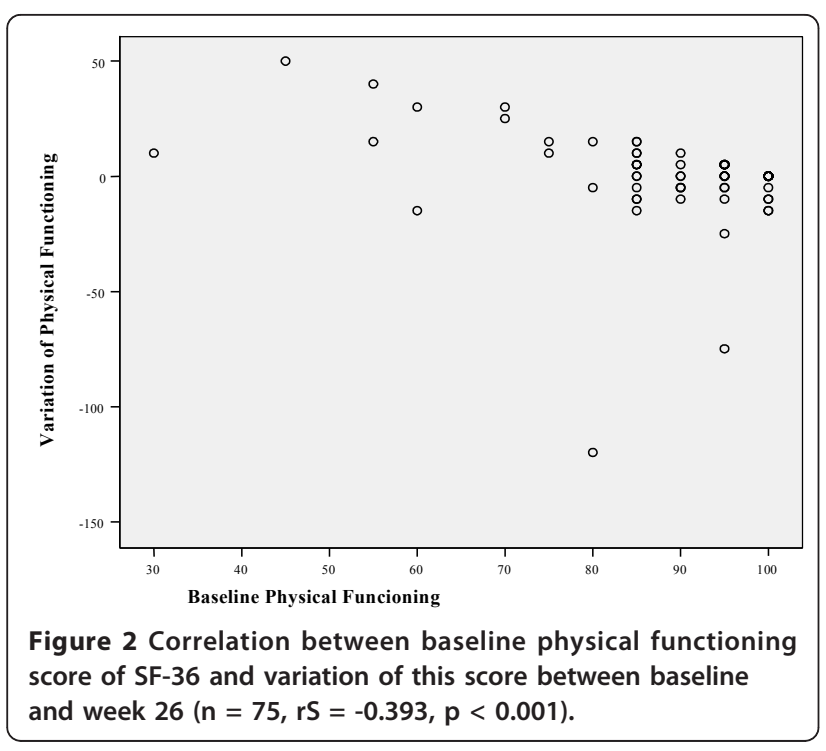

improvements in some aspects of quality of life, suggesting that educational interventions improve workers' health conditions. Further studies are necessary to determinate the value of education on long term outcomes and in populations at higher risk of WMSD.

\section{Authors' contributions}

ACS was the principal investigator, and participated in the protocol development, study design, conduct of the study, analysis of the data, writing up and revision of drafts of the manuscript. KFR participated in the program conducted for the control group, and VAA participated in data organization. MB participated in the analysis of data, writing up and revision of drafts of the manuscript. RMX participated in the study design, coordination and conduct of the study.

All authors read and approved the the final manuscript.

\section{Competing interests}

The authors declare that they have no competing interests.

Received: 13 July 2010 Accepted: 28 January 2011

Published: 28 January 2011

\section{References}

1. Bureau of Labor Statistic BLS, U: Workplace injuries and illnesses. News 2009 [http://www.bls.gov/iif/home.htm], [cited 2009; Technical information].

2. INSS/Dataprev: Anuário Estatístico da Previdênica Social.Edited by: MPS/ DATAPREV/INSS. Brasília, BRASIL; 2007.

3. Sim J, Lacey RJ, Lewis M: The impact of workplace risk factors on the occurrence of neck and upper limb pain: a general population study. BMC Public Health 2006, 6:234.

4. Helliwell PS, Taylor WJ: Repetitive strain injury. Postgrad Med J 2004, 80(946):438-43.

5. Malchaire JB, et al: Musculoskeletal complaints, functional capacity, personality and psychosocial factors. Int Arch Occup Environ Health 2001, 74(8):549-57.

6. van Tulder M, Malmivaara A, Koes B: Repetitive strain injury Lancet 2007, 369(9575):1815-22.

7. Werner RA, et al: Predictors of upper extremity discomfort: a longitudinal study of industrial and clerical workers. J Occup Rehabil 2005, 15(1):27-35.

8. Yeng TL: Avaliação de um programa educacional multidisciplinar em pacientes com distúrbios ósteo-musculares relacionados ao trabalho (DORT). Universidade de São Paulo: São Paulo, Brasil; 2002.
9. Ege SC: Consulting in industry: Moving beyond traditional interventions. Work 2006, 26(3):243-50.

10. Peper E, Gibney KH, Wilson VE: Group training with healthy computing practices to prevent repetitive strain injury (RSI): a preliminary study. Appl Psychophysiol Biofeedback 2004, 29(4):279-87.

11. Buckle P: Ergonomics and musculoskeletal disorders: overview. Occup Med (Lond) 2005, 55(3):164-7.

12. Daltroy $L H$, et al: $A$ controlled trial of an educational program to prevent low back injuries. N Engl J Med 1997, 337(5):322-8.

13. Brox Jl, et al: Systematic review of back schools, brief education, and fear-avoidance training for chronic low back pain. Spine J 2008, 8(6):948-58.

14. IJzelenberg $\mathrm{H}$, Meerding WJ, Burdorf A: Effectiveness of a back pain prevention program: a cluster randomized controlled trial in an occupational setting. Spine 2007, 32(7):711-9.

15. Pillastrini $P$, et al: Evaluation of two preventive interventions for reducing musculoskeletal complaints in operators of video display terminals. Phys Ther 2007, 87(5):536-44.

16. Frost $P$, Haahr JP, Andersen $\mathrm{JH}$ : Reduction of pain-related disability in working populations: a randomized intervention study of the effects of an educational booklet addressing psychosocial risk factors and screening workplaces for physical health hazards. Spine 2007, 32(18):1949-54.

17. Rempel DM, et al: A randomised controlled trial evaluating the effects of two workstation interventions on upper body pain and incident musculoskeltal disorders among computer operators. Occupational and Environmental Medicine 2006, 63(5):300(7).

18. Greene BL, DeJoy DM, Olejnik S: Effects of an active ergonomics training program on risk exposure, worker beliefs, and symptoms in computer users. Work 2005, 24(1):41-52

19. Gerr F, et al: A randomised controlled trial of postural interventions for prevention of musculoskeletal symptoms among computer users. Occup Environ Med 2005, 62(7):478-87.

20. Melhorn JM: A prospective study for upper-extremity cumulative trauma disorders of workers in aircraft manufacturing. J Occup Environ Med 1996, 38(12):1264-71.

21. Abramson JH: WINPEPI (PEPI-for-Windows): computer programs for epidemiologists. Epidemiol Perspect Innov 2004, 1(1):6.

22. da Mota Falcao D, Ciconelli RM, Ferraz MB: Translation and cultural adaptation of quality of life questionnaires: an evaluation of methodology. J Rheumatol 2003, 30(2):379-85.

23. de Soarez PC, et al: Translation into Brazilian Portuguese and validation of the Work Limitations Questionnaire. Rev Panam Salud Publica 2007, 22(1):21-8.

24. Devereux K, Robertson D, Briffa NK: Effects of a water-based program on women 65 years and over: a randomised controlled trial. Aust $J$ Physiother 2005, 51(2):102-8.

25. Andersen $L L$, et al: $A$ randomized controlled intervention trial to relieve and prevent neck/shoulder pain. Med Sci Sports Exerc 2008, 40(6):983-90

26. Blangsted AK, et al: One-year randomized controlled trial with different physical-activity programs to reduce musculoskeletal symptoms in the neck and shoulders among office workers. Scand J Work Environ Health 2008, 34(1):55-65.

27. Lied TR, Kazandjian VA: A Hawthorne strategy: implications for performance measurement and improvement. Clin Perform Qual Health Care 1998, 6(4):201-4.

\section{Pre-publication history}

The pre-publication history for this paper can be accessed here: http://www.biomedcentral.com/1471-2458/11/60/prepub

doi:10.1186/1471-2458-11-60

Cite this article as: Santos et al.: Impact on the Quality of Life of an Educational Program for the Prevention of Work-Related Musculoskeletal Disorders: a randomized controlled trial. BMC Public Health 2011 11:60. 